\title{
FORMACIÓN ONLINE: MÉTODO ABIERTO BASADO EN NÚMEROS (ABN) Y APLICACIÓN PRÁCTICA EN ALUMNADO DE 10 A 14 AÑOS
}

\author{
María del Carmen Canto López \\ Departamento de psicología, Universidad de Cádiz \\ mari.cantolopez@uca.es \\ Malena Manchado Porras \\ Departamento de psicología, Universidad de Cádiz (UCA) \\ malenamp97@gmail.com \\ José Carlos Piñero Charlo \\ Departamento de didáctica (área de matemáticas), Universidad de Cádiz \\ josecarlos.pinero@gm.uca.es \\ Manuel Aguilar Villagrán \\ Departamento de psicología, Universidad de Cádiz \\ manuel.aguilar@uca.es \\ Estibaliz Aragón Mendizábal \\ Departamento de psicología. Universidad de Cádiz \\ estivaliz.aragon@uca.es \\ Cándida Delgado Casas \\ Departamento de psicología. Universidad de Cádiz \\ candida.delgado@uca.es
}

Recepción Artículo: 18 mayo 2021 Admisión Evaluación: 18 mayo 2021 Informe Evaluador 1: 22 mayo 2021 Informe Evaluador 2: 27 mayo 2021 Aprobación Publicación: 02 junio 2021

Proyecto I+D+i. Ref. PID2019-105584GB-I00 del Ministerio español de Ciencia e Innovación.

\section{RESUMEN}

En la actualidad, uno de los mayores retos educativos sigue siendo superar las dificultades que genera el aprendizaje matemático en el alumnado de Educación Primaria y Educación Secundaria. Recientemente, se ha incrementado la investigación sobre el aprendizaje y la cognición matemática debido a las consecuencias personales y sociales del fracaso enel aprendizaje. Una de las líneas de trabajo enfatiza la conveniencia de realizar intervenciones que incorporen los procesos cognitivos implicados en el aprendizaje de las matemáticas, y hagan uso de estrategias innovadoras como el método de cálculo Abierto Basado en Números (ABN). El presente estudio pretende analizar el impacto del programa formativo online para la implantación del método ABN en cursos altos de Educación Primaria y primer ciclo de Educación Secundaria Obligatoria. El proyecto se distribuye en cuatro fases: (1) Selección de escuelas; (2) Programa formativo online para docentes; (3) Estudio empírico para analizar la efectividad del método ABN; y (4) Transferencia de resultados y diseño de materiales y APP educativas matemáticas. Se presentan los resultados obtenidos en la primera etapa, con el objetivo de analizar el 


\section{FORMACIÓN ONLINE: MÉTODO ABIERTO BASADO EN NÚMEROS (ABN) Y APLICACIÓN PRÁCTICA EN ALUMNADO DE 10 A 14 AÑ̃OS}

impacto del programa formativo y la incorporación de materiales manipulativos para diversos contenidos matemáticos. Los participantes del programa formativo pertenecen a centros educativos de la provincia de Cádiz y fueron seleccionados incidentalmente. La muestra está compuesta por 40 participantes (19 investigadores, 3 docentes expertos en ABN, 18 docentes y 3 estudiantes de máster en Educación Secundaria). Se presentan los resultados extraídos tras la formación del profesorado y las prácticas de aula llevadas a cabo, los cuales muestran la necesidad de formación del profesorado para la incorporación de dinámicas alternativas en los cursos altos de Educación Primaria. Se espera abrir nuevas líneas de investigación basadas en la implementación de programas y talleres para la aplicación de estrategias innovadoras en los centros educativos.

Palabras clave: método abn; aprendizaje matemático; materiales manipulativos; formación profesorado, App

\section{ABSTRACT}

On line training: the open calculation based on numbers (abn) method in 10-14 aged students. Currently, one of the greatest challenges are the difficulties generated by mathematical learning in primary and secondary school students. In recent years, research on mathematical learning and cognition has increased due to the personal and social consequences of failure in mathematics learning. One of the researching topics emphasizes the convenience of carry out classroom interventions incorporating the cognitive processes involved in mathematical learning, and making use of innovative strategies such as the open calculation based on numbers (ABN). The current study aims to analyze the impact of an online training program for the implementation of the ABN methodology in the third cycle of Primary Education and the first two academic years of Secondary Education. The project is divided into four stages: (1) Schools' choice; (2) Online teachers training program; (3) Empirical study to analyze the effectiveness of the ABN method; and (4) Transfer of results and design of materials and mathematics educational APP. The results obtained in the first stage are presented below, analyzing the impact of the training program and adding manipulative resources by several mathematical contents. The participants of the training program belong to the School system form Cádiz province and were incidentally selected. The sample is composed of a total of 40 participants (19 researchers, 3 expert teachers in ABN, 18 teachers and 3 master's degree students in Secondary Education). The results of the teacher training and classroom practices show the need for teacher training for the incorporation of alternative dynamics in the upper grades of Primary Education. It is expected to open new research lines based on the implementation of programs and workshops for the implementation of innovative strategies in schools.

Keywords: ABN method; mathematical learning; manipulative resources; teacher training, APP

\section{INTRODUCCIÓN}

En la actualidad, el rendimiento académico en matemáticas en muchos países europeos muestra un amplio margen de mejora, aunque España muestra unos resultados similares a evaluaciones anteriores (PISA, 2018; TIMMS, 2019). Esto último es relevante dadas las consecuencias personales y sociales de las dificultades en el aprendizaje matemático, así como por la importancia de esta área de conocimiento en el crecimiento económico (Aguilar, Aragón y Navarro, 2015). Por ello, es necesario que el alumnado que se encuentran en Educación Infantil (EI) y Educación Primaria (EP) alcancen un nivel de destreza en la materia que les prepare para las etapas escolares superiores y, finalmente, para ser competentes a la hora de afrontar la vida adulta (European Commission, 2016).

Así, aunque aún no se conoce con exactitud cómo funciona el proceso de aprendizaje de la matemática, parece conveniente atender a los procesos cognitivos implicados en el mismo, con el fin de reducir la brecha entre alumnado con y sin dificultades (Aguilar, et al., 2015). En este sentido, desde los enfoques cognitivos del aprendizaje de la matemática se promueve que los métodos de enseñanza conduzcan a una compresión profunda de los contenidos. La recomendación desde la perspectiva cognitiva es ligar dichos contenidos a la resolución de problemas significativos e interesantes para el alumnado, permitiéndoles construir su propio conocimiento, y contar con sus experiencias reales como punto de partida (Bruning, Schcraw, y Norby, 2012). Además, el hecho 
de que tanto alumnado como profesores verbalicen los procesos y estrategias empleados y reflexionen sobre los mismos, hace posible el desarrollo de capacidades estratégicas en los estudiantes, evitando que se centren en encontrar la solución sin entender el proceso, y al mismo tiempo facilitando la detección de errores en los razonamientos matemáticos. Para ello, se pueden utilizar recursos como los debates en el aula, los trabajos en grupo, las presentaciones orales de asuntos matemáticos, etc. De esta manera, la perspectiva cognitiva hace hincapié en la necesidad de diversificar e intercalar los distintos tipos de problemas planteados al alumnado, así como las formas de presentarlos, para así fomentar su capacidad de discriminación y su flexibilidad a la hora de abordar las tareas y decidir cómo y cuándo usar determinados procedimientos. Finalmente, este enfoque destaca la importancia de utilizar métodos que promuevan la autoeficacia de los estudiantes y su actitud positiva hacia la materia. Por supuesto, para lograr un enfoque de instrucción que cumpla con todas las recomendaciones anteriores sobre experiencias en el aula, es necesario que los docentes tengan una formación sólida y específica en matemáticas y en los métodos para enseñar la materia (Bruning, Schcraw, y Norby, 2012). Además, otro elemento que podría ser beneficioso sería el uso de las nuevas tecnologías (Zhang, Trussell, Gallegos, y Asam, 2015) y de métodos de enseñanza alternativos e innovadores, como el método abierto basado en números (ABN) (Cerda, Aragón, Pérez-Wilson, Navarro, y Aguilar, 2018). Este método pretende romper con los mecanismos memorísticos y alejados de la realidad que implica el sistema tradicional, empleando una metodología más abierta y cotidiana, en la que los problemas u operaciones tienen distintas soluciones válidas y el alumnado aprende a interiorizar los conceptos matemáticos mediante materiales manipulativos (Diaz-López, Torres y Lozano, 2017). Además, otorga la posibilidad de adaptar el aprendizaje al progreso individual de cada alumno, fomentando así la motivación y la actitud positiva hacia la materia (Martínez-Montero y Sánchez, 2019).

En este sentido, se destacan recursos de enseñanza-aprendizaje que encajan con una visión metodológica de este tipo. En primer lugar, resaltar las herramientas novedosas basadas en nuevas tecnologías para el aprendizaje de la matemática en educación infantil y primeros cursos de educación primaria. Dentro de estos recursos, destacar las APPs para la mejora del sentido numérico, que abordan tareas de comparación de magnitudes, subitización, hechos numéricos y estimación en la recta numérica, y al mismo tiempo suponen un estímulo atractivo para los estudiantes (Mera, et al., 2019). En segundo lugar, en Educación Primaria, existen distintas propuestas didácticas que buscan un aprendizaje a través del juego y los materiales manipulativos, creando un ambiente distendido donde el alumnado desarrolla interés por la materia y aprende en función de su propio ritmo (Lara, 2017; Fraile, 2017). Dentro de este tipo de propuestas en Educación Primaria, algunas recurren a nuevas tecnologías como la interfaz gráfica de Matlab Guide, que permite explicar conceptos y métodos numéricos mediante visualización gráfica, e incluye la posibilidad de experimentar interactivamente mediante elementos manipulables virtuales (Gatica y Ares, 2012). Otras proponen un itinerario detallado para el aprendizaje matemático, en este caso de fracciones, compuesto por actividades correspondientes a contextos informales: recursos cotidianos, manipulativos y juegos; contextos intermedios: un recurso literario y otro tecnológico; y contextos formales: un recurso gráfico (Alsina y Franco, 2020). En esta misma línea, en el trabajo de García-Moya, Blanco, y Fernández-Cézar (2019) se realizaron talleres dirigidos a estudiantes con alto rendimiento matemático, compuestos por materiales manipulativos, pero incluyendo además lo que denominan "retomáticos", es decir, actividades de tipo reto matemático diseñadas por el propio estudiante. En este caso, los materiales manipulativos se dividían en estructurados y no estructurados. Los primeros son aquellos diseñados con el fin de construir un aprendizaje (bloques lógicos, las regletas de Cuisenaire y los materiales de construcción de poliedros mediante caras), mientras que los segundos, no han sido elaborados con ese fin (depresores, la baraja española y los dados de colores).

Finalmente, las investigaciones citadas convergen en la idea de los beneficios que proporcionan las metodologías innovadoras y flexibles que emplean materiales manipulativos y elementos motivadores para el alumnado. En consecuencia, se destaca la importancia de asegurar la calidad de la enseñanza matemática a través de una formación efectiva del profesorado en métodos alternativos, como la metodología de cálculo Abierto 


\section{FORMACIÓN ONLINE: MÉTODO ABIERTO BASADO EN NÚMEROS (ABN) Y APLICACIÓN PRÁCTICA EN ALUMNADO DE 10 A 14 AÑ̃OS}

basado en números (ABN). Este método alternativo, se caracteriza por su carácter abierto y el tratamiento que lleva a cabo sobre números y cantidades, dichas características propician el desarrollo de estrategias de cálculo mental y el razonamiento matemático. Al tratarse de un método abierto, el alumnado puede llegar al resultado de formas muy diferentes y no existe una única forma de resolver los algoritmos escritos o las tareas planteadas. Por este motivo, se puede decir que el método ABN se adapta a cada alumno/a, en función de su ritmo de aprendizaje, dominio y estrategias de cálculo (Canto López et al., 2019). Por último, destacar la importancia en la resolución y formulación de problemas de forma contextualizada, con apoyo de referentes, en los que el alumnado debe apoyarse para la resolución de los mismos. El método ABN está siendo implantado en los centros educativos desde el curso 2008/2009. Actualmente, más de 200.000 escolares (alrededor de 8000 grupos-clase) están aprendiendo matemáticas con este procedimiento (Aragón et al, 2017) en España.

\section{OBJETIVOS DE LA INVESTIGACIÓN}

El presente trabajo tiene como objetivo general analizar los resultados derivados de la primera etapa del proyecto, es decir, el programa formativo online dirigido a docentes, que aborda la implantación del método abierto basado en números (ABN) en las aulas de segundo y tercer ciclo de Educación Primaria (EP) y primer ciclo de Educación Secundaria Obligatoria (ESO).

Los objetivos específicos son los siguientes:

1. Registrar el impacto de las sesiones de formación online del programa formativo, solicitando a los docentes el diseño de, al menos, una sesión ABN que refleje los contenidos impartidos.

2. Analizar las aportaciones de los docentes para observar el grado de implicación derivado de la formación.

3. Estudiar el contenido de la/s sesión/es propuestas por los docentes, describiendo y analizando los contenidos matemáticos abordados en las mismas (cálculo mental, descomposición factorial, potencias y raíces).

4. Examinar los materiales manipulativos que se proponen en dichas sesiones, así como la puesta en práctica de los contenidos diseñados.

\section{PARTICIPANTES}

La muestra total está compuesta por 40 participantes, de los cuales 16 son investigadores en el área de la cognición y didáctica de las matemáticas, 3 son docentes expertos en ABN, 18 son docentes (13 de escuelas de España y 5 de escuelas chilenas) y 3 son estudiantes de máster en Educación Secundaria (MAES). Los participantes mencionados son aquellos que han asistido a las cuatro sesiones formativas sobre el método ABN realizadas hasta la fecha. No obstante, los datos que se registran en el presente trabajo se obtuvieron de una parte de esa muestra total: la parte docente, compuesta por 13 participantes de los centros educativos seleccionados. Los 7 maestros de educación primaria y 5 profesores de matemáticas de educación secundaria son los participantes centrales, ya que son los que proporcionaron los datos analizados en los próximos apartados. Estos pertenecen a centros educativos de la provincia de Cádiz y fueron seleccionados incidentalmente teniendo en cuenta el propósito del presente trabajo en relación con la metodología ABN.

\section{METODOLOGÍA}

Para el presente trabajo se empleó una metodología basada en el registro y análisis de datos a nivel descriptivo. El proyecto l+D+i (PID2019-105584GB-I00 del Ministerio español de Ciencia e Innovación), dentro del cual se enmarca el presente estudio, está compuesto por 4 fases, tal y como se recoge en la figura 1. Hasta el momento se han llevado a cabo las dos primeras fases: selección de centros participantes y el programa formativo online, el cual se inició en noviembre de 2020 y finaliza en mayo de 2021. 
Figura 1. Fases de trabajo del proyecto I+D+i (PID2019-105584GB-100)

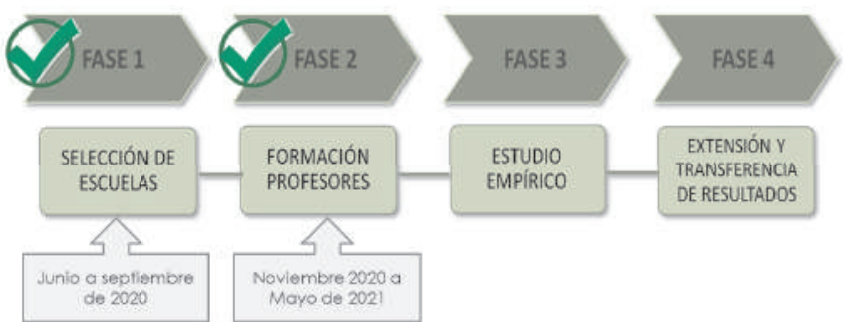

En primer lugar, antes del comienzo del programa online formativo, el profesorado participante rellenó una encuesta inicial sobre aspectos generales y específicos de la docencia, el conocimiento y el uso de la metodología ABN. De los 18 docentes de EP y ESO, 12 completaron la encuesta a través del enlace proporcionado en el campus virtual. La encuesta se compone de 14 preguntas. En la segunda fase se ha llevado a cabo el programa online formativo, el cual consta de 6 sesiones sobre diversos contenidos comunes a segundo y tercer ciclo de EP y primer ciclo de ESO: operaciones y cálculo mental, descomposición factorial, potencias y raíces, porcentajes e inicio al álgebra. En las diferentes sesiones el profesorado experto en metodología ABN expuso las dinámicas de trabajo, secuencias y materiales que utilizan para trabajar dichos contenidos en el aula. En el campus virtual se han facilitado recursos y documentos que complementan las sesiones online y tienen a su disposición foros de consulta y debate.

Dos meses antes de finalizar el programa online formativo, se solicitó a los participantes que diseñaran una sesión ABN y la llevaran a cabo con su alumnado, abordando en la misma alguno de los contenidos trabajados en las sesiones formativas sobre el método ABN. Esa aplicación práctica fue entregada por algunos docentes EP y profesores de ESO, que pudieron aplicar algunos de los contenidos trabajados durante las sesiones online. Dichos participantes enviaron sus sesiones prácticas a través de una entrega en el campus virtual, en formato PDF, con fotos o enlaces para explicitar lo trabajado en el aula. Cada sesión práctica incluía en el mismo archivo dos bloques básicos: el primero, la descripción de la sesión o sesiones que se hayan llevado a cabo; el segundo, fotografías de los materiales empleados y de la puesta en práctica del contenido de la sesión. Los docentes y profesores comenzaron a llevar a cabo las sesiones en los dos últimos meses del programa formativo. Tras esa primera fase de registro, se procedió a analizar las contribuciones de los docentes de forma descriptiva. Por un lado, en cuanto a los datos cuantitativos, se analizó el porcentaje de participación docente en la actividad. Por otro lado, en cuanto a los datos cualitativos, se examinaron dos cuestiones: Ios contenidos concretos abordados en cada una de las sesiones (cálculo mental, descomposición factorial, potencias y raíces e inicio al álgebra), y los materiales empleados para poner en práctica dichos contenidos. Finalmente, tras analizar los datos cuantitativos y cualitativos, se procedió a redactar los resultados y a realizar la discusión de los mismos, así como las conclusiones finales.

\section{RESULTADOS}

Tal y como se ha comentado previamente, en un primer momento, se solicitó al grupo de docentes y profesores la realización de una encuesta inicial. De los 12 docentes y profesores que completaron la encuesta, fueron 5 hombres (41,7\%) y 7 mujeres (58,3\%). Al inicio de la formación el $91,7 \%$ de los participantes conocían el método $A B N$ y de los que conocían el Método ABN, el 58,3\% no había realizado ninguna formación referente al método $A B N$.

La aplicación práctica en el aula fue llevada a cabo 3 docentes de EP (en un aula de $4^{0}$ de EP y dos de $6^{0}$ de EP) y una profesora de primero de ESO. Los contenidos trabajados en las sesiones fueron cálculo escrito a través de la resolución razonada de problemas, descomposición factorial, potencias y raíces e inicio al álgebra. A 


\section{FORMACIOÓN ONLINE: MÉTODO ABIERTO BASADO EN NÚMEROS (ABN) Y APLICACIÓN PRÁCTICA EN ALUMNADO DE 10 A 14 AÑ̃OS}

continuación, se expone el análisis y desarrollo de las diferentes sesiones, especificando contenidos trabajados, material utilizado y ejemplificaciones llevadas a cabo por el alumnado.

\section{Aula $4^{\circ}$ Educación Primaria.}

Este grupo pertenece a un centro público y llevaron a cabo cuatro sesiones en las que se trabajaron los siguientes contenidos: cálculo escrito y resolución razonada de problemas, descomposición avanzada e inicio al álgebra. A continuación, se presenta una breve descripción de cada una de las sesiones.

Sesión 1. Personalización del cálculo escrito. A través de la utilización del algoritmo escrito abierto que proporciona el Método ABN, el alumnado personaliza la forma de resolver las operaciones, realizando los pasos que mejor se adapte a su ritmo de aprendizaje (figura 2). En el ejemplo, se presenta el algoritmo escrito de la resta por detracción, en la que se refleja el manejo de las cantidades por parte del estudiante. En dos pasos se ha resuelto la operación y utiliza de una forma avanzada la estrategia de redondeo (8.172 - 1. 173 y 6.999 - 512), fomentando la personalización del proceso por parte del alumnado.

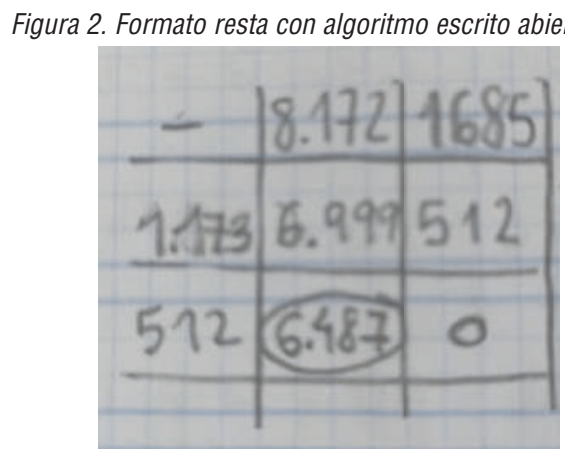

Sesión 2. Resolución razonada de problemas. Dentro de un contexto de situación-problema planteado, el alumnado trabaja con decimales utilizando el algoritmo abierto de la división (figura 3). El problema planteado fue el siguiente: Tenía 67,30€ ahorrados. Me he comprado 6 camisetas. ¿Cuánto me ha costado cada camiseta? ¿cuánto me ha sobrado?

Figura 3. Formato división con algoritmo escrito abierto $A B N$

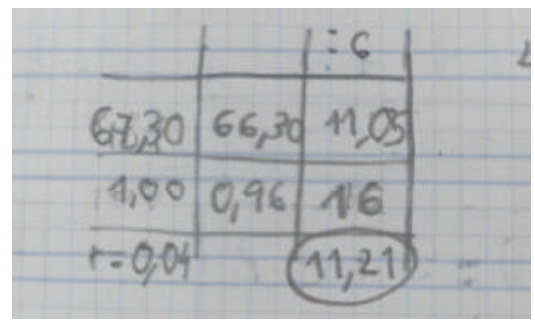

En el ejemplo presentado, se desarrolla el formato escrito de división por reparto. En este caso el estudiante refleja un manejo excelente de los repartos con cantidades decimales, aspecto que se ve beneficiado por el planteamiento de la situación-problema en un contexto de dinero, en el que previamente se ha manejado las monedas para propiciar la experimentación del alumnado.

Sesión 3. Descomposición avanzada y cálculo mental. En esta sesión el alumnado debía resolver la descomposición de forma "creativa", utilizando tres operaciones diferentes para llegar al número dado, 4,20 (figura 4). 
Figura 4. Descomposición creativa (sol de números)

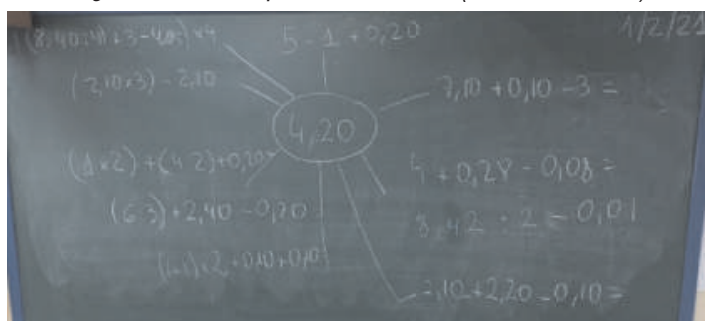

La actividad resulta en la pizarra, muestra una tarea denominada "sol de números", en la que partiendo de una cantidad dada en el círculo central, en este caso 4,20, el alumnado debe verbalizar las diferentes opciones para poder conseguir dicha cantidad utilizando al menos dos operaciones, combinando tres cantidades. En el ejemplo, se muestra el dominio del cálculo mental utilizando las cuatro operaciones trabajas en Educación Primaria (suma, resta, producto y división).

Sesión 4. Iniciación de las ecuaciones con balanza. En esta sesión, se utilizó como material manipulativo una balanza (figura 5). En una parte de la balanza ponemos una cantidad y en la otra una menor. El alumnado debe experimentar con el material, para llegar a equilibrar los dos lados de la balanza con las piezas amarillas, que pueden ser individuales 0 repetidas como el caso de la imagen. La ecuación presentada es $20=2 x+2$ y en la balanza se presenta en la parte izquierda dos piezas en el 10, por lo que tenemos 20; y en la otra parte, un ficha en el dos. Se ofrece al alumnado dos fichas que debe colocar en una misma cantidad, para obtener el doble de esta cantidad (2x). El alumnado debe experimentar con las piezas dadas y colocarlas en la cantidad que nos equilibre la balanza $(20=2+2 * 9 ; 20=2+18 ; 20=20)$.

Figura 5. Iniciación a las ecuaciones con balanza

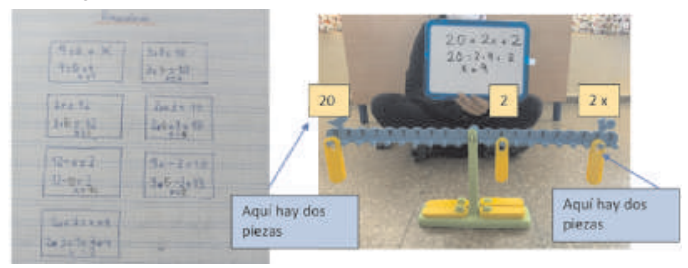

\section{Aula $6^{\circ}$ de Educación Primaria}

Esta aula pertenece a un centro público. Llevaron a cabo cuatro sesiones, trabajando los siguientes contenidos: números primos y compuestos, descomposición factorial, cálculo del mcm y MCD. Las sesiones se desarrollaron de la siguiente manera:

Sesión 1. Criba de Eratóstenes, números primos y compuestos. En la presente sesión el alumnado inició el procedimiento de obtención de los números primos menores de cien con la técnica de la criba de Eratóstenes, se trabajaron los conceptos de múltiplos, divisores, primos y compuestos y se repasaron los criterios de divisibilidad.

El material utilizado para la criba de Eratóstenes fue la tabla del 100 y para las descomposiciones de números compuestos se utilizaron bloques, en los que cada color representaba un número primo (figura 6). En el ejemplo se muestra la criba de Eratóstenes en la tabla del 100, quedando sin marcar los números primos y tapados con el tapón los números compuestos que aparecen en varias tablas de multiplicar del 2 al 12 . También se muestra la descomposición del 108, tres bloques amarillos y dos bloques verdes $(3 \times 3 \times 3 \times 2 \times 2 ; 27 \times 4=108)$. 
Figura 6. Criba de Eratóstenes y descomposiciones números compuestos

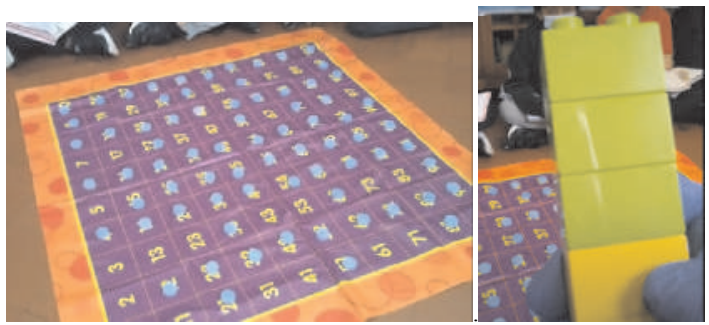

Sesión 2. Criterios de divisibilidad y descomposición factorial números primos y compuestos. En esta sesión el alumnado continúo el trabajo iniciado en la primera sesión, practicando y repasando los criterios de divisibilidad y la descomposición factorial de números compuestos utilizando los bloques. El alumnado debía reconocer y diferenciar entre múltiplos y divisores, identificar y justificar números primos y compuestos, usar la tabla del 100 para encontrar y mostrar los números primos así como las regularidades numéricas en la tabla, aplicar los criterios de divisibilidad, realizar la descomposición factorial de manera manipulativa. y en el cuaderno a través de ramaje, barra vertical o de manera horizontal.

Se continuó utilizando la tabla del 100 y los bloques para facilitar la descomposición factorial.

Sesión 3. Inicio al cálculo del mínimo común múltiplo y máximo común divisor. En esta tercera sesión el alumnado aprende a hallar el máximo común divisor y el mínimo común múltiplo a partir de la descomposición factorial, utilizando el material manipulable, y aplicarlo a la resolución de problemas (figura 7). En este caso, se sigue utilizando como apoyo en la descomposición factorial, la representación con los bloques. En el ejemplo presentado se debe hallar el máximo común divisor (MCD) de 210 y 462 . Se realiza la descomposición con apoyo del material manipulativo. El 210 se representa con un bloque violeta, otro azul, uno verde y otro amarillo

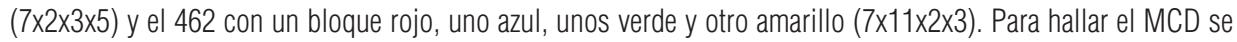
cogen los bloques del mismo color, los que se repiten el menor número de veces, por lo que el resultado es $7 \times 2 \times 3$, siendo igual a 42.

Figura 7. Cálculo del máximo común divisor con bloques.

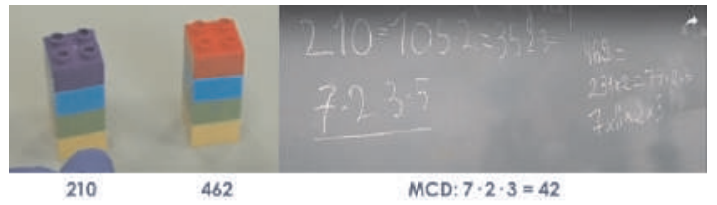

Sesión 4. Desarrollo de juego Scracht sobre divisibilidad y descomposición factorial. En esta sesión y con apoyo de la especialista en TIC del centro educativo, el alumnado desarrolló dos juegos utilizando la aplicación Scratch para la práctica de los contenidos trabajados. En la figura 8 se muestra el ejemplo de los dos juegos desarrollados por el alumnado para que puedan repasar los conceptos trabajados por medio de las nuevas tecnologías que tan atractivas resultan para el alumnado de esta etapa educativa. 
Figura 8. Juegos Scratch sobre divisibilidad y descomposición factorial
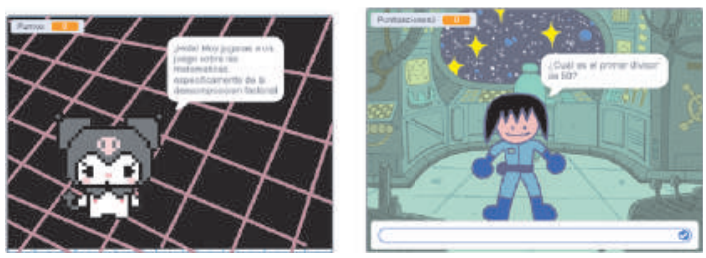

Aula $6^{\circ}$ de Educación Primaria

El grupo pertenece a un centro educativo privado. En este caso se llevaron a cabo tres sesiones en las que se trabajaron los contenidos de potencias y raíces. A continuación, se explica brevemente las tres sesiones desarrolladas por el docente.

Sesión 1. Inicio de potencias y raíces. En esta primera sesión se presentó las diversas situaciones de las potencias y raíces cuadradas, se practicó el cálculo mental de los cuadrados de los números del 1 al 12 y la raíz cuadrada de los cuadrados de los números del 1 al 12. Las situaciones planteadas son las siguientes:

En mi jardín hay 5 macetas que contienen 5 rosas cada una.

3 camiones van cargados con 3 contenedores, cada contenedor alberga 3 cajas y cada caja contiene 3 televisores.

Mi salón es cuadrado y está solado con 36 baldosas cuadradas.

En el Jardín Botánico hay un bosque en el que hay tantas hileras de árboles como árboles tiene cada hilera. En total hay 144 árboles.

Sesión 2. Propiedades de las potencias. En esta sesión se trabajó la construcción y cálculo de potencias de base 10 y su expresión en notación científica.

Sesión 3. Técnica de los cuadrados. El alumnado practicó la técnica de los cuadrados, mediante la resolución razonada de situaciones-problema, como el siguiente: El tablero de ajedrez tiene 8 filas y es un cuadrado. ¿Cuántas casillas tiene? Si en lugar de llegar a la letra H, Ilegara a la letra L, ¿cuántas casillas tendrá? El material utilizado fue la representación del cuadrado para poder resolver el problema planteado, tal y como se presenta en la figura 9 .

Figura 9. Técnica de los cuadrados para la resolución de problemas.

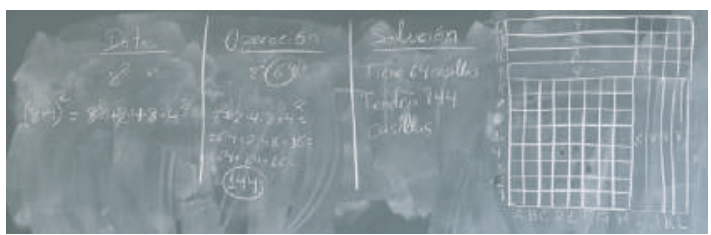

En la figura anterior se presenta la representación gráfica de la técnica de los cuadrados para facilitar el cálculo de un cuadrado mayor a uno dado. En este caso se debe pasar del cuadrado de 8, que sabemos que es 64 , al cuadrado de 12, que se haya añadiendo filas y columnas a la representación gráfica del cuadrado de 8 , debiendo conseguir un nuevo cuadrado más grande, en este caso con 12 filas y 12 columnas.

Aula $1^{\circ}$ de ESO

Esta aula pertenece a un centro. Los contenidos trabajados en este caso son las potencias y raíces. En este grupo se llevaron a cabo dos sesiones:

Sesión 1. Concepto de cuadrados de dígitos menores a 10. En esta primera sesión se trabajó el concepto de cuadrado y la representación con material manipulativo de los diferentes cuadrados. El material utilizado fue construido por la docente, utilizando cartulinas de colores y un panel con bolsillos (figura 10). Con el material 
manipulativo se construyeron los diferentes cuadrados que se presentan en la pizarra. Con este recurso el alumnado visualiza y manipula la construcción de los cuadrados, de manera que se interioriza y razona el concepto de cuadrado, posteriormente servirá de base para hallar las raíces cuadradas.

Figura 10. Representación y cálculo de cuadrados

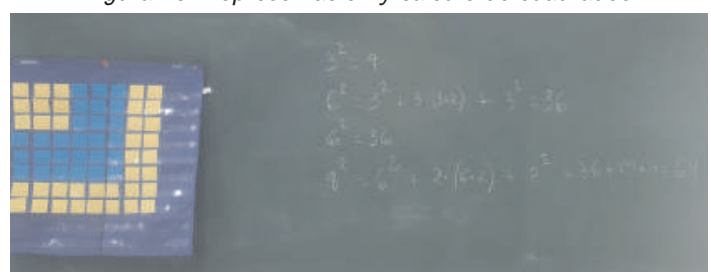

Sesión 2. Cuadrado de decenas y semidecenas, técnica de los cuadrados. En la segunda se sesión, se trabajó el cálculo de cuadrados de decenas y semidecenas, además se utilizó el mismo material de apoyo para trabajar la técnica de los cuadrados (práctica del paso de cuadrado menor a un cuadrado mayor) y el cálculo de raíces a partir de la técnica trabajada y utilizando el formato $\mathrm{ABN}$ y tradicional del algoritmo escrito para las raíces (figura 11). En el método $A B N$, el algoritmo escrito de la raíz cuadrada se presenta en formato de rejilla o tabla, en la que se van representando las diferentes cantidades que se van hallando a través de la representación del cuadrado, teniendo como base el cuadrado de las decenas completas. En el ejemplo mostrado, se parte del cuadrado de 50 y se pasa al cuadrado de 53 , insertando tres filas, tres columnas y nueve baldosas para completar la representación del cuadrado.

Figura 11. Resolución de raíces cuadradas con técnica de los cuadrados.

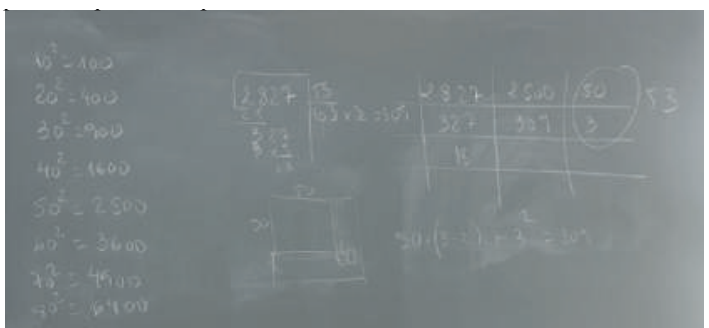

\section{DISCUSIÓN}

Tras el análisis de las sesiones llevadas a cabo por el profesorado participante y a pesar, de una baja participación, las muestras presentadas parecen indicar que el programa formativo online, ha facilitado el uso de materiales manipulativos en los cursos altos de EP y primer curso de ESO. Igualmente, se ha continuado la base cálculo y resolución razonada de problemas en diversas áreas del currículum de matemáticas, tal y como se muestra en las prácticas de aula llevadas a cabo por parte del profesorado. Una vez finalizado el programa formativo, se analizará las causas de la baja participación del profesorado participante en la aplicación práctica en el aula, y se propiciará el desarrollo de estas experiencias en el próximo curso, de manera que podamos incluir un análisis más completo del programa formativo.

Destacar la posibilidad de ofrecer un mejor conocimiento del método ABN en los niveles educativos mencionados anteriormente y facilitar la transición a ESO del alumnado participante en el proyecto. Durante el programa formativo online se han afianzado las relaciones entre los docentes de EP y el profesorado de ESO, compartiendo dificultades y aspectos que deben ser tenidos en cuenta durante la transición de etapas educativas. 
Por todo lo anterior, se reafirma la necesidad de una formación sólida y de calidad para los docentes y profesores de matemáticas, para poder aplicar de forma efectiva al aula los métodos alternativos e innovadores de enseñanza de la matemática, abordando los componentes cognitivos del proceso de aprendizaje matemático y permitiendo al alumnado alcanzar una comprensión profunda y razonada de los conceptos a través de experiencias más flexibles y conectadas con la realidad.

\section{CONCLUSIONES}

La forma de enseñanza está cambiando vertiginosamente, poco tiene que ver cómo se enseñaba o se aprendía hace diez años con la forma de ahora. Los docentes y profesores de matemáticas deben adaptarse a los cambios, estar actualizados con la información, la legislación, los métodos alternativos de enseñanza y ser conscientes de la necesidad de utilizar metodologías abiertas para atender la diversidad dentro del aula. Es por ello, que este tipo de programas formativos deben continuar para proporcionar una formación sólida en relación a las matemáticas.

Debemos tener en cuenta que el estudio presentado es el resultado preliminar de un programa formativo que continuará a través de la plataforma educativa de la UCA, con el objetivo de afianzar las relaciones establecidas con los centros participantes, propiciar una mayor aplicación de los contenidos trabajados en el aula, y así poder finalizar las demás fases del proyecto. A partir de este estudio, se hace necesario profundizar en el análisis sobre la repercusión de programas formativos en metodologías alternativas y los posibles perfiles didácticos del profesorado de matemáticas. Estos perfiles docentes podrían estar relacionados con la actitud y el desempeño del docente 0 profesor en relación a la forma de trabajar con el alumnado, forma de llegar los contenidos más avanzados al alumnado de 10 a 14 años, tipo de recursos que se utilizan, utilización de nuevas tecnologías, entre otras.

Una de las principales limitaciones de este estudio ha sido no poder implementar el

programa formativo de forma presencial, como consecuencia de la crisis sanitaria ocasionada por la Covid19 en España, y las restricciones asociadas a las misma. Así pues, debe ser considerado ampliar la formación en el método ABN, en los cursos altos de EP y primer ciclo de la ESO, para prevenir las posibles dificultades y reducir así los riesgos que conlleva la transición a esta etapa escolar. Este programa formativo online se podrá llevar a cabo en posteriores años para continuar apoyando la formación del profesorado especialista en matemáticas.

\section{REFERENCIAS BIBLIOGRÁFICAS}

Aguilar, M., Aragón, E. y Navarro, J.I. (2015). Mathematical learning disabilities (MLD). State of art. Revista de Psicología y Educación, 10(2), 13-42. http://www.revistadepsicologiayeducacion.es/pdf/125.pdf

Alsina, Á., y Franco, J. (2020). Promoviendo la educación matemática inclusiva desde el Enfoque de los Itinerarios de Enseñanza de las Matemáticas: el caso de las fracciones. APEduC Revista-Investigação e Práticas em Educação em Ciências, Matemática e Tecnologia, 1(2), 13-29. https://apeducrevista.utad.pt/index.php/apeduc/article/view/130/28

Bruning, R.H., Schcraw, G.J., y Norby, M.M. (2012). Psicología cognitiva y de la instrucción. Madrid: Pearson Educación (pp. 351-353). Canto López, M.C., Aguilar, M., García-Sedeño, M.A., Navarro, J.I., Aragón, E., Delgado, C. y Mera, C. (2019). Numerical Estimation and Mathematical Learning Methodology in Preschoolers. Psychological reports, 124 (2), 438-458. doi: 10.1177/0033294119892880

Cerda, G., Aragón, E., Pérez-Wilson, C., Navarro, J.I., y Aguilar, M. (2018). The Open Algorithm Based on Numbers (ABN) method: An effective instructional approach to domain-specific precursors of arithmetic development. Frontiers in Psychology, (9), 1811. doi: 10.3389/fpsyg.2018.01811

Díaz-López, M., Torres, N., y Lozano, M. C. (2017). Nuevo enfoque en la enseñanza de las matemáticas, el método ABN. Revista INFAD de Psicología. International Journal of Developmental and Educational Psychology., 3(1), 431-434. doi: 10.17060/ijodaep.2017.n1.v3.1012 
European Commission (2016). Mathematics for Europe. Retrieved March 4, 2019, from https://ec.europa.eu/futurium/en/system/files/ged/finalreport_maths.pdf

Fraile, M. (2017). Matemáticas, materiales manipulativos y rutinas. Proyecto para segundo de Primaria (tesis de pregrado). Universidad Internacional de La Rioja, La Rioja, España.

García-Moya, M., Blanco, R., y Fernández-Cézar, R. (2019). Matemáticas manipulativas y alto rendimiento. En M., García-Moya. (Ed.), Inclusión, Tecnología y Sociedad. Investigación e Innovación en Educación (pp.22632277). Castilla-La Mancha, España: DYKINSON.

Gatica, S. N., y Ares, 0. E. (2012). La importancia de la visualización en el aprendizaje de conceptos matemáticos. Edmetic, 1(2), 88-107. doi: 10.21071/edmetic.v1i2.2853.

Lara, R. (2017). Enseñanza de la estadística utilizando el juego y materiales manipulativos como recurso didáctico en 6o de Primaria (tesis de pregrado). Universidad Internacional de La Rioja, La Rioja, España.

Martínez-Montero, J., y Sánchez, C. (2019). [Enrichment of mathematical learning in preschool and primary school with the $A B N$ method]. Madrid, ES: Pirámide.

Mera, C., Ruiz, G., Aguilar, M., Aragón, E., Delgado, C., Menacho, I., Marchena, E., García, M. \& Navarro, J.I. (2019). Coming Together: R\&D and children's entertainment company in designing apps for learning early Math. Frontiers in Psychology, 9, 2751. doi: 10.3389/fpsyg.2018.02751

PISA (2015). Results in focus. OECD. Retrieved March 4, 2019, from https://www.oecd.org/pisa/pisa-2015results-in-focus.pdf

Zhang, M., Trussell, R.P., Gallegos, B., y Asam, R.R. (2015). Using math apps for improving student learning: An exploratory study in an inclusive fourth grade classroom. Tech Trends, 59(2), 32-39. doi: 10.1007/s11528015-0837-y 